\title{
Application of Collagen Implants in Achilles Tendon Injuries
}

\section{Oryan $\mathbf{A}^{1^{*}}$, Alidadi $\mathbf{S}^{1}$ and Moshiri $\mathbf{A}^{2}$}

${ }^{1}$ Department of Pathology, School of Veterinary Medicine, Shiraz University, Shiraz, Iran

${ }^{2}$ Razi Drug Research Center, Iran University of Medical Sciences, Tehran, Iran

\begin{abstract}
Achilles tendon injuries are common and complex especially in athletes and active people. Healing process occurs via scar tissue formation leading to poor outcomes for patients. Current surgical and non-surgical treatment modalities lead to sub-optimal tendon healing and each of them have complications. Therefore, to repair and restore tendon structure and function, a tendon graft is needed; therefore autografts, allografts and xenografts have been used. However, each of these grafts is associated with several limitations such as donor site morbidity and pain, poor biocompatibility, disease transmission and immune reaction which lead to graft rejection and failure. Tissue engineering is an advancing field that can either augment surgical repair or provide an alternative method for Achilles tendon repair. Among the components of tissue engineering technologies, the present article has discussed scaffolds and emphasized on collagen-based biomaterials. This article illustrated the causes behind the essential need for tissue engineering in Achilles tendon repair. Furthermore, it described the current literature regarding the use of collagen in Achilles tendon repair and the main points regarding application of collagen biomaterials and scaffolds in tendon tissue engineering.
\end{abstract}

Keywords: Achilles tendon; Healing; Tissue engineering; Scaffold; Collagen

\section{Introduction}

The incidence of Achilles tendon rupture is increasing and about $75 \%$ of all ruptures occur during sport activities particularly among racket sports [1,2]. Tendon healing is an important and hot topic in orthopedics and orthopedic sports medicine [3]. The treatment of Achilles tendon is performed surgically using either standard open or mini-invasive (percutaneous) technique and/or non-surgically through different mobilization substitutes [3-5]. Surgical treatment is recommended in highly active sport persons because of earlier and safer return to sports $[1,2]$. This kind of treatment may lead to shorter rehabilitation period and safer return to sport player [4]. Various immobilization methods such as casting, functional bracing with or without weight-bearing and range of motion training have been designed in managing tendon injuries $[4,5]$. These modalities are associated with limitations including tendon adhesion, infection and the risk of tendon re-rupture; therefore there is a need for another promising method to facilitate the healing process of the Achilles tendon defects $[1,2]$. There are some concerns with tendon grafts, including autografts, allografts and xenografts, because of their wellknown disadvantages [3,6-9]. Tissue engineering as a multidisciplinary approach introduces and suggests some solutions to enhance tendon healing and regeneration $[3,10,11]$. Of the most appropriate scaffolds used in the field of tendon tissue engineering are collagen-based constructs [10]. Many collagen structures alone or in combination with other bioactive molecules and cells have been applied in tendon healing $[10,12,13]$. Therefore, the purpose of this study was to review the treatment techniques of Achilles tendon injuries to explain how tissue engineering can help in this field. Moreover, we clarified the advantages and disadvantages of the collagen-based structures, and explained some collagen characteristics and properties with some recent studies.

\section{Structure and function of tendon}

Tendons are considered as dense fibrous connective tissues that connect muscles to bones and facilitate joint movement $[3,10,14,15]$. Generally, tendons are formed dominantly from type I collagen (85\% of dry weight) embedding in a proteoglycan-water matrix [6]. Collagen is produced by fibroblasts and tenocytes. After production of the collagen molecules as procollagens within the cells, they are secreted out of the cell and converted into tropocpllagen [3]. The tropocollagen molecules are cross-linked and aggregated to microfibrils that form collagen fibrils. Aggregation of numerous collagen fibrils form a collagen fiber as the basic and the smallest unit of a tendon that is visible by light microscopy. Bundles of collagen fibers form fiber bundles and then fascicles that form tertiary fiber bundles $[3,5,10]$. These architectures of tendon are covered by a loose connective tissue named endotenon containing blood and lymphatic vessels and nerves [10]. The large fiber bundles and tertiary fiber bundles with endotenon are surrounded by another connective tissue called epitenon. Finally, all these structures together are covered by a connective tissue layer namely the paratenon or the synovial sheath that sometimes together with the epitenon are called the peritenon or peritedineum $[3,10,16]$. Tendons are attached to bones and muscles by a type III collagen-rich fibrocartilaginous tissue named enthesis and myotendinous junction, respectively [5]. The collagen proteins are responsible for mechanical properties of tendons because of their high tensile strength [14]. In addition to tendon cells and collagens especially type I collagen ( $85 \%$ of dry matter) and smaller amounts of other collagens including collagens types III, V, XII and $\mathrm{XI}$, tendons are composed of water, proteoglycans, glycoproteins and elastic fibers $[6,14,16]$.

\section{Achilles tendon injuries}

The Achilles or calcaneal tendon is considered as the most prone to rupture tendon in body [2,17]. It is the largest and strongest tendon in human body [2]. Most sports-related injuries in tendons occur in this tendon. Tendinopathies affecting the Achilles tendons are related to two locations including the mid portion and the insertion site of the tendon onto the calcaneus $[4,17,18]$. The Achilles tendon injuries are relatively frequent in young and active people and in most instances occur during

*Corresponding author: Professor Ahmad Oryan, Department of Pathology, School of Veterinary Medicine, Shiraz University, Shiraz, Iran, Tel: +98 7112286950; Fax: +98 7112286940; E-mail: oryan@shirazu.ac.ir

Received September 26, 2015; Accepted October 28, 2015; Published November 03, 2015

Citation: Oryan A, Alidadi S, Moshiri A (2015) Application of Collagen Implants in Achilles Tendon Injuries. J Sports Med Doping Stud 5: 169. doi:10.4172/21610673.1000169

Copyright: $\odot 2015$ Oryan A et al. This is an open-access article distributed under the terms of the Creative Commons Attribution License, which permits unrestricted use, distribution, and reproduction in any medium, provided the original author and source are credited. 
sports activities such as racket games, football, and handball as well as in running athletes and in the age groups of 30-39 years old associated with repetitive movements $[2,18]$. Three mechanisms of injury include a sudden pushing off from weight-bearing forefoot with the extended knee (sprint starts, racket sports and jumping), unexpected dorsiflextion of the ankle (slipping into a hole or falls), and violent dorsiflextion of a plantar-flexed foot (falling from height) [2,17].). Intrinsic factors such as age, sex, biomechanics, presence or absence of systemic diseases and genetic factors may be involved in tendon injuries. On the other hand, extrinsic factors including physical load and trauma also may be stimulant $[1,2,18]$. Administration of steroids or fluoroquinolones and previous rupture of the contra lateral tendon are some risk factors for rupture of Achilles tendon [4]. Other possible factors include tendon degeneration, poor vascularity of the tendon and hyperthermia induced by exercise [2]. Tendon injuries occur most commonly in male individuals than females [1].

\section{Tendon healing}

Healing of tendon is a complex process in which many factors are involved. In fact, the process is interaction of several elements including blood cells, tissue cells, growth factors, cytokines, inflammatory mediators, hormones and so on $[8,10,19]$. Tendon healing is composed of three phases including inflammatory, proliferative and remodeling phases. At the moment of injury, a hematoma is formed and an inflammatory reaction is initiated $[3,10]$. During this phase, leukocyte cells, erythrocytes and platelets are present in the blood clot and initiate further inflammatory and angiogenesis processes [3]. The blood clot, necrotic tissue and debris in the injured are phagocytized by the inflammatory cells. Platelets release several molecules which regulate and progress the process. This phase lasts 24 hours to five days [20].

During the second phase, the fibroblasts are the predominant cells; they produce collagen molecules dominantly type III collagen and proteoglycans and a temporary stability is provided. This stage lasts from five days to a few weeks $[3,10,20]$. The third phase can be divided to consolidation and maturation phases. Cellularity and tissue hydration decrease, collagen type III is replaced with collagen type I and the collagen fibers start to align along the line of stress during this phase $[3,10]$. At the end of the consolidation phase or beginning of the maturation phase, the hierarchical organization of the tendon starts to develop and the small-sized, unimodal collagen fibrils differentiate to larger fibrils and form a bimodal and then multimodal fibrils' pattern $[3,10,18]$. The fibrils are then assembled to fibers, fiber bundles and fascicles. These developmental changes result in more weight bearing capability and higher physical activity $[18,20]$. In fact, these morphological and physical changes are responsible in enhancing the biomechanical properties of the injured tendon during its long term remodeling phase $[10,15,18]$. Tendon healing is complicated when the tendon injury is severe and the defect size is large [3,10]. In such cases, treatment is associated with more limitations such as adhesion of the injured area to the surrounding tissues and muscle disturbance such as muscle necrosis, fibrosis and atrophy; in such instances the outcome is poor [19]. Because of low vascularity and cellularity and minimum metabolic rate in tendons, the healing process is slow and normal structural hierarchy and biomechanical performance similar to those of the intact tendon may never be achieved [18,19,21]. Treatment and management of tendon injuries remains a clinical challenge for orthopedic medicine.

\section{Current treatment methods of tendon injuries}

Diagnosis of a tendon rupture is based on the clinical examination and confirming a palpable gap at the ruptured site and a positive
Thompson's or Simmonds' test $[1,2,4]$. Because of pain and weakness especially in the chronic injuries, the patients are unable to weight bear on the affected limb $[4,5,18]$. The early diagnosis can be confirmed by ultrasonography or magnetic resonance imaging $[1,4]$. It is important that the patients with Achilles tendon rupture return to their pre-injury level of sport activity $[1,2,18]$. The purpose of treatment is to shorten the immobilization period and return the patients sooner to sports $[4,5]$. Conservative treatments including rest, corticosteroid therapy, ultrasound, laser therapy or shockwave relieve pain and it is likely to fail $[1,2]$. Treatment can be divided into non-surgical (cast or splint immobilization or functional bracing) or surgical (open, minimally invasive or percutaneous) approaches $[1,2,4]$. Both surgical and nonsurgical treatment regimes exhibit advantages and disadvantages. It seems the surgical procedures can reduce the risk of re-rupture in Achilles tendon compared to the non-surgical treatment methods $[1,4,18]$. In fact, non-surgical treatment lowers re-rupture rate to 10 $12 \%$ compared to less than $3 \%$ in surgical treatment [4]. However, it has been stated that the risk of other complications is higher with operative methods $[1,2]$. Complications other than re-rupture include skin complications such as wound infection, skin or tendon necrosis and draining sinus formation, scarring and fibrous adhesions at the wound site, and disturbed sensation $[1,2,4,18,20]$. Compared with percutaneous and minimally invasive surgery methods, open surgery is associated with higher complication, higher costs, but lower re-rupture rate $[1,2,17]$. Although it is accepted that surgical therapy should be performed for young and active patients versus conservative or nonsurgical treatment for the elders [2]. Optimal treatment for Achilles tendon injuries or rupture remains controversial. Cast immobilization or functional bracing after the initial intervention can accelerate rehabilitation [1].

Regarding the cases of severe injuries, an option for replacing damaged tendons is biological grafts including autografts, allografts and xenografts $[3,21,22]$. However, these grafts were not associated with satisfactory outcomes because of their drawbacks including high morbidity and pain at the donor site, need to further surgery and limited availability for autografts. Additionally, the risk of disease transmission and tissue rejection are the disadvantages with allografts and xengrafts $[3,10]$. Furthermore, with the exception of autografts that provide all tenogenic, tenoinductive and tenoconductive properties, other grafts have no tenogenisity or even tenoinductivity $[3,10,20]$.

\section{Tissue engineering}

Despite all efforts to improve the treatment quality, alternative strategies are required to treat tendon injuries. To achieve such promising clinical progress, a wide knowledge from different sciences including medicine, biomechanics, biochemistry, biology, nanotechnology and engineering is required [10]. Given the limitations associated with the treatment methods of tendon injuries, one helpful and promising option may be the use of tissue engineering modalities [16,20,23]. Tissue engineering is regarded as a promising option for managing large tendon defects having three different constituents including tissue scaffolds, factors promoting healing and cells or stem cells $[3,10]$. The most important part of tissue engineering field is scaffold because of its crucial function. In fact, the therapeutic value of an engineered tissue without a suitable scaffold but containing the two other elements is low [10]. A scaffold should provide an appropriate environment for cell attachment, migration, differentiation and proliferation as well as matrix remodeling and regeneration [24]. Scaffolds should have several properties including biodegradability, biocompatibility and bioactivity. Moreover, they should be porous to facilitate cell migration and proliferation and movement of growth factors. They should have 
suitable mechanical properties and could be constructed in a threedimensional mode $[2,10]$. Porosity is regarded as a fundamental characteristic that provides space for cell adhesion and migration and allows transport of nutrients and metabolic discharge [10]. An ideal tissue-engineered scaffold should have at least $70 \%$ porosity and pore size ranging from tens to hundreds micrometers to provide a suitable three-dimensional space to facilitate cell attachment and proliferation and promote regeneration of the new tissue [12]. However, high porosity may be associated with reduced mechanical strength.

Scaffolds are classified on the basis of different variables; for instance, based on the source, the scaffolds are divided into three main categories including biologic (natural), synthetic (artificial) and hybrid scaffolds $[3,10,24]$. Biologic scaffolds are produced by biologic molecules such as collagen, gelatin, fibrin, alginate, elastin, chitosan, hyaluronic acid, demineralized bone matrix, chondroitin sulfate and so on $[10,24]$. Despite all advantages, the biologic scaffolds exhibit poor mechanical properties. The synthetic scaffolds are associated with higher mechanical properties and lower biocompatibility compared to the natural scaffolds [24]. These polymers include polylactic, polypropylene, polyglycolic acid, polydioxanone, polycaprolacton, nylon, carbon fibers, calcium phosphates, hydroxyapatite and ceramics $[3,10,24]$. Hybrid scaffolds are constructed by a combination of biological and synthetic scaffolds to overcome the well-known limitations when are used alone [24]. Among these scaffolds, collagen is very important from many points of view, since it constitutes the most frequent protein component in the extracellular matrix (ECM) of many soft and hard connective tissues of mammals [10].

\section{Collagen and tendon healing}

Collagen is a favorite material for tendon regeneration since it is the major ECM component in tendons. Several studies have demonstrated the applicability of different forms of collagen in tendon healing [2528]. Collagen has well-documented structural, physical, chemical and immunological properties including high availability, easy purification from living organisms, low antigenicity, non- or low-cytotoxicity, good hemostatic and cell-binding properties as well as biological plasticity [10,29]. Nevertheless, several disadvantages of collagenbased scaffolds such as cost, and the possibility of immunogenic and disease transmission risks, should be considered when are used as a tendon substitutes [20]. In addition, some other properties of collagen such as high cost of pure type I collagen, variation in fiber sizes, low elasticity, poor mechanical strength, poor dimensional stability because of swelling, and possible antigenic reaction may adversely affect its application $[10,19,30]$. Collagen has high biodegradability character so that it can undergo degradation upon implantation within four to five weeks [31]. It has been stated that collagen membrane could prevent tendon adhesion following surgical treatment of the ruptured Achilles tendon in rat [32]. Moreover, the tensile strength and expression of collagen type I were improved in the animals which were treated by the collagen membrane.

Collagen can be applied in various forms including films, gels, sponges, membranes, nanoparticles, scaffolds or powder [30-32]. As an example, Geistlich Bio-Gide ${ }^{\bullet}$ is a resorbable collagen membrane with a bilayer structure consisting of natural collagen of pig without further cross-linking or chemical additives with a porous and dense surface [31]. There are several collagen-based grafts derived from different sources such as small intestinal sub-mucosa (SIS) of porcine jejunum, urinary bladder membrane, pericardium or dermis $[3,10,14]$. To form such membranes, the non-collagenous components are removed and the natural collagen structure is retained [20]. Some commercially available collagen materials approved by the Food and Drug Administration (FDA) for tendon repair include Restore ${ }^{\mathrm{TM}}$ (from SIS, not cross-linked), and CuffPatch (from SIS, cross-linked with carbodiimide), GraftJacket ${ }^{\mathrm{TM}}$ (human acellular dermal matrix, not-cross-linked), TissueMend (bovine dermis and bovine dermal ECM), Zimmer collagen repair patch (porcine dermis cross-linked with diisocyanate), OrthADAPT ${ }^{\mathrm{TM}}$ (equine pericardium), Permacol (porcine acellular dermal matrix) and Bio-Blanket ${ }^{\mathrm{TM}}[10,14,19,30,33]$. Two main issues with collagen include the low strength provided by collagen and its high degradation that is not desired for an ideal delivery system or carrier [34]. To overcome these issues and having a sustained release of growth factors, some researchers have fabricated the delivery systems to express lower degradation rate so that the scaffold can retain the growth factors for a long time at the implantation site [27,28,34]. Some researchers have cross-linked the collagen fibers with other biomaterials to enhance their biomechanical properties [35,36]. Others have incorporated some compounds to biomaterials and loaded in scaffolds to immobilize growth factors and prolong their retention at the defect site $[37,38]$.

\section{Cross-linking and biomechanical properties of collagen}

The naturally cross-linked collagen fibrils in the structure of skin, tendons and bones lead to rigidity of these organs [35,39]. These fibrils are made and stabilized by forming covalent bonds between four to eight collagen molecules. Three $a$-chains are interlaced in the collagen triple-helix to form the collagen molecules [35]. The triplestranded collagen helix (Triple-helix) is composed of repeated Gly-X-Y sequences and is stabilized by intra- and inter-chain hydrogen bonds. It should be noted that $\mathrm{X}$ and $\mathrm{Y}$ are largely proline and hydroxyproline, respectively [35,39]. Telopeptides made of 15 to 26 amino acids mostly lysine and hydroxylysine residues are the very short $\mathrm{N}$ - and C- terminal regions that do not participate in triple-helix formation [35]. The total Gly-Pro-Hyp sequence content is considered as one of the most important factors influencing collagen thermostability [35].

One of the major limitations of collagen-based scaffolds in tendon engineering technologies is their low biomechanical properties [10]. Physical or chemical cross-linkers are expected to improve the biomechanical performance of the engineered tendons $[10,39,40]$. It has been demonstrated that the mechanical properties of collagen can be improved by cross-linking process [39-41]. For this purpose, a variety of reagents and methods such as chemical cross-linkers such as aldehydes like gluteraldehyde (GTA), formaldehyde, diisocyanates such as hexamethylenediisocyanate, carbodiimides such as 1-ethy-3(3-dimethylaminopropyl) carbodiimide, and acyl azides and physical cross-linking by the use of dehydrothermal treatment, ultraviolet and gamma irradiation have been employed $[2,10,39,41]$. These physical methods increase the resistance to the collagenolytic degradation [39]. Although UV-irradiation may induce the free radicals formation, it can improve the mechanical strength of the material [39]. In addition to amino groups, GTA react with carboxylic, amides and other protein groups $[39,40]$.

There are some concerns about cross-linking of the collagenbased structures with GTA including cytotoxicity due to releasing the monomer from the glutaraldehyde polymer and calcification of the collagen-based material as well [39-41]. However, GTA reduces the immunogenicity and increases the resistance of the collagenous scaffold to enzymatic degradation $[39,41]$. Cross-linking with formaldehyde prolongs the absorption and reduces the tensile strength of collagenbased scaffolds [39,41]. Moreover, there is the potential of toxicity and adverse reactions due to the brittleness of scaffolds cross-linked with 
formaldehyde [39]. All other cross-linking agents are associated with cytotoxicity and this consequence limits their application [40]. Given these limitations, effective and non-toxic cross-linkers such as genipin, a natural iridoid glycoside derived from the fruit extract of the gardenia plant (Gardenia jasmindides Ellis), have been used and approved. Genipin has been applied for gelatin films and hydrogels [40,41]. Genipin cross-links gelatin through the epsilon amino group present in lysine and hydroxylysine residues [41].

Another method for enhancing the collagen affinity to growth factors is the application of polysaccharides such as heparin that have a high binding affinity with many proteins such as bone morphogenetic proteins (BMPs) and growth factors present in platelets such as vascular endothelial growth factor (VEGF) and basic fibroblast growth factor (bFGF) [36,38]. Hannink et al. [38] stabilized growth factors and protected them from proteolytic degradation for long-term delivery of BMP-2, by covalently incorporating heparin into the cross-linked collagen coated tri-calcium phosphate-hydroxyapatite granules. Wenpo et al. [36] added pectin, an anionic plant polysaccharide hydrogel, to collagen-hydroxyapatite composite and obtained desirable mechanical properties, low cytotoxicity and suitable enzyme degradation rate. Pectin is involved in the mechanical and morphological development of plant tissues and it has been considered as an interesting option for tissue engineering approaches such as a carrier for drug delivery and an implant coating [42]. In fact, polysaccharides such as heparin and pectin interact with proteins rich in hydroxyproline including collagen via covalent bond $[36,38,41]$.

The combination of different materials is also a strategic design for achieving hybrid scaffolds. For instance, Kwon et al. [43] fabricated an artificial Achilles tendon comprising a braided silk tube and lyophilized collagen sponge that improved regeneration of neo-tendon tissues in rabbits. The structure and tensile strength of these neo-tendons were similar to those of the native tendon. In fact, collagen alone has poor physical properties and it cannot bear the mechanical load of the materials; therefore, the silk tube scaffold compensated these limitations.

\section{Collagen degradation}

Since collagen is the main structural protein in the body, it is resistant to neutral proteases [39]. Only specific collagenases which are metalloproteinases requiring calcium can cleave the triple helix from the $\mathrm{N}$-terminus. In addition, elastases and cathepsins involve in proteolysis of collagen fibers through digesting the non-helical regions $[10,39]$. When the collagen molecules are administrated in tissue engineering technologies, different cellular responses occur depending on the shape of collage material [10,39]. For example, collagen gels are penetrated by fibroblasts to contract the gel, while fibroblasts, macrophages and neutrophils penetrate to the surrounding structures to degrade the collagen [39]. It has been stated that the collagen films without crosslinking are degraded during two to seven weeks. However, absorption and degradation can be manipulated by crosslinking methods.

\section{Antigenicity, immunogenicity and sterilization}

Collagen is known for its excellent biocompatibility and poor immunologic reactions. Nevertheless, since most of the collagenbased structures are derived from animal tissues, their implantation may be associated with immune reaction of the host and even rejection $[3,10]$. In fact, since the main source of collagen is the tissues harvested from porcine or bovine skins, tendons or bones, the risk of allergic reactions and transmission of zoonotic disease such as bovine spongiform encephalopathy (BSE) or transmissible spongiform encephalopathy (TSE) must be noticed [10,39]. To avoid this problem, acellularization modalities can eliminate the immunogenicity and enhance incorporation of the collagen-based scaffolds $[10,24,44]$. These methods remove the nuclear and cytoplasmic antigens, while the ECM structure and mechanical properties of the scaffold are maintained [10]. Enzymatic, physical and chemical methods have been used for this purpose including sodium dodecyl sulfate, Triton X-100, $\mathrm{NaCL}$, trypsin, ethylene diamine tetraacetic acid (EDTA), and tributyl phosphate $[10,24,44,45]$. Vavken et al. [45] introduced Triton $\mathrm{X}$ as the most effective solution for acellularization of the porcine anterior cruciate ligament. However, in a study conducted by Elder et al. [44], 2\% SDS was the most efficient concentration for cartilage acellularization. In addition, glutaraldehyde as a chemical cross-linker can reduce, but does not eliminate the antigenicity of the scaffolds of the collagen sources $[10,39]$. Despite such theoretical concerns, collagen seems safe and demonstrates low antigenicity when it is used as an implantable and injectable biomaterial [39]. Among moist heat, dry heat, ethylene oxide and $\gamma$-irradiation, the last one has been considered as the most reliable method in sterilizing the collagen biomaterials so that a dose of $2.5 \mathrm{Mrad}$ has been used for complete sterilization [39]. However, $\gamma$-irradiation can decrease the mechanical strength of collagen; therefor it makes the material sensitive to collagenase degradation $[10,39]$.

\section{Collagen-based scaffolds with cells and growth factors}

To optimize the benefits of treatment methods of Achilles tendon defects, it may be advantageous to seed the scaffolds with stem cells to support healing and remodeling processes and enhance the potential of migration of the mesenchymal cells and their differentiation into tenocytes and tenoblasts $[1,46,47]$. Different forms of collagen structures have been used alone or in combination with other bioactive molecules, growth factors alone or in the form of platelet-rich plasma (PRP) or stem cells [10]. The ECM from porcine SIS is a biomaterial that can recruit stem cells to participate in the tendon healing. Zhang et al. [47] combined type I collagen matrix with weft-knitted silk-poly (lactide-co-glycolide) mesh scaffold and seeded this structure with mesenchymal stem cells and enhanced Achilles tendon repair in rabbit. Pietschmann et al. [46] showed that polyglycolic acid in combination with collagen type I scaffolds seeded with mesenchymal stem cells may be less effective than the scaffolds loaded with tenocytes. Sixteen weeks after repair, the Achilles tendon of rat treated with scaffolds seeded with tenocytes had better structural performance and mechanical properties than those treated with scaffolds seeded with stem cells or controls.

Since stem cells differentiate and integrate into tendon, the combination of scaffolds and cells can promote maturation of collagen fibers and result in enhanced tendon repair [10,47]. It should be highlighted that if the degradation rate of the scaffold is too rapid, it will lead to loss of the scaffold as the cell support and result in formation of an unorganized granulation tissue formation [39,47]. On the other hand, slow rate of degradation of the material may accumulate it for a longer time in the defect area so that there is not enough space for tissue regeneration. In addition, such material behaves as a foreign material and negatively affects tissue regeneration [47].

In addition to stem cells, addition of tenocytes to collagen-based scaffolds has been represented as a valuable source of cells for tendon tissue engineering purposes [13]. It should be paid attention that an optimal cell-to-collagen ratio can improve the delivery of stem cells to the healing tendon, while application of higher ratio of mesenchymal cells has not been found beneficial $[13,46]$. The collagen gels exhibit superior cell-seeding efficiency, while the collagen sponges present 
greater mechanical strength so that a combination of collagen gels with collagen fibers or sponges represents a promising strategy [14]. Martinello et al. [48] fabricated a re-cellularized collagen gel scaffold using adipose-derived mesenchymal stem cells in treatment of tendon injuries. This scaffold resulted in an enhanced expression of collagen type I production in the ECM.

The healing and regeneration processes are affected and regulated by many factors such as metalloproteinase, growth factors and inflammatory mediators at different times during the healing processes $[3,10,24,34]$. These factors play important roles in tendon healing by regulating cellular migration, proliferation, differentiation and maturation as well as production of extracellaular matrix [3,10,34]. However, the growth factors such as BMPs are very expensive that limits their exogenous application on tendon healing [39]. Therefore, some researchers successfully embedded the platelet-rich plasma as a costeffective source of several growth factors within the tissue-engineered collagen-based implants to promote the healing and regeneration of large Achilles tendon defects in rabbits [25,26,28].

\section{Future Directions and Conclusion}

Another promising approach in application of collagen-based constructs is development of collagen nano-particles in the field of drug delivery carriers $[14,39]$. Some advantages of such nanoparticles include sustained release, reduced side effects, improved bioavailability and drug protection [14]. For the drug-loaded collagen systems, it must be paid attention to the drug because of its effects on the cellular reactions for collagen degradation [39]. For example, corticosteroids can decrease degradation of collagen, whereas the immunostimulants or growth factors increase the degradation rate [39]. Electrospinning that is a unique method in which the scaffolds are fabricated exhibit high porosity and micro- to nano-scale topography similar to the natural ECM structure, has received considerable attention as a possible processing technique for fabrication of the scaffolds used in tendon tissue engineering $[3,10,14]$. Xu et al. [12] fabricated a 3D aligned nanoyarn collagen-based scaffold, using electrospinning technology that provides sufficient space for cell adhesion and infiltration with desirable mechanical properties in comparison to the random nanofiber and aligned nonofiber scaffolds. The materials in collagen solution are formed into nano- and micro-sized continuous fibers by electrospinning and the fibers are in uni-directional and parallel alignment $[12,27]$. The electrospun nanofibrous scaffolds can be used to improve cell attachment and increase cellular activity [12,27,49]. However, this technology has limitations such as availability of electrospinning devices, poor biomechanical properties of the collagenbased constructs, cell-interaction with biomaterials and cost in respect to $3 \mathrm{D}$ structures $[14,18]$.

In conclusion, advantages of collagen including good biocompatibility and biodegradability, suitable porosity especially in sponge forms, and ability to enhance cellular penetration all reflect the potential of collagen in the growing field of tissue engineering. These factors make collagen suitable for scaffolds fabrication to delivery growth factors or cells for enhancing the healing and repair of Achilles tendon injuries. However, it should be noticed that the disadvantages mentioned above such as fast biodegradation of collagen implants, high cost of pure type I collagen preparation can limit further application of collagen implants. Other disadvantages include hydrophilicity resulting in swelling and rapid release of its contents, and poor biomechanical strength. In addition, variability of the isolated collagen in terms of fiber size, cross-linking density and fiber alignment, variability in enzymatic degradation rate and the risk of zoonotic disease transmission with collagen derived from bovine sources are other limiatations. Therefore; given these explanations, the use of collagen-based scaffolds and biomaterials particularly in clinical studies remains challenging.

\section{References}

1. Jones MP, Khan RJ, Carey Smith RL (2012) Surgical interventions for treating acute achilles tendon rupture: key findings from a recent cochrane review. J Bone Joint Surg Am 94: e88.

2. Jiang N, Wang B, Chen A, Dong F, Yu B (2012) Operative versus non-operative treatment for acute Achilles tendon rupture: a meta-analysis based on current evidence. Int Orthp 36: 765-773.

3. Moshiri A, Oryan A (2013) Tendon and ligament tissue engineering, healing and regenerative medicine. J Sports Med Doping Stud 3: 126.

4. Soroceanu A, Sidhwa F, Aarabi S, Kaufman A, Glazebrook M (2012) Surgica versus nonsurgical treatment of acute Achilles tendon rupture: a meta-analysis of randomized trials. J Bone Joint Surg Am 94: 2136-2143.

5. Nourissat G, Berenbaum F, Duprez D (2015) Tendon injury: from biology to tendon repair. Nat Rev Rheumatol 11: 223-233.

6. Thomopoulos S, Parks WC, Rifkin DB, Derwin KA (2015) Mechanisms of tendon injury and repair. J Orthop Res 33: 832-839.

7. Oryan A, Goodship AE, Silver IA (2008) Response of a collagenase-induced tendon injury to treatment with a polysulphated glycosaminoglycan (Adequan). Connect Tissue Res 49: 351-360.

8. Oryan A, Silver IA, Goodship AE (2010) Metrenperone enhances collagen turnover and remodeling in the early stages of healing of tendon injury in rabbit. Arch Orthop Trauma Surg 130: 1451-1457.

9. Oryan A, Moshiri A, Meimandi-Parizi A (2014) In vitro characterization of novel tissue engineered based hybridized nano and micro structured collagen implant and its in vivo role on tenoinduction, tenoconduction, tenogenesis and tenointegration. J Mater Sci Mater Med 25: 873-897.

10. Oryan A, Moshiri A, Sharifi $P$ (2012) Advances in injured tendon engineering with emphasis on the role of collagen implants. Hard Tissue 1: 12.

11. Moshiri A, Oryan A (2012) Role of tissue engineering in tendon reconstructive surgery and regenerative medicine: current concepts, approaches and concerns. Hard Tissue 1: 11

12. Xu Y, Wu J, Wang H, Li H, Di N et al. (2013) Fabrication of electrospun poly(Llactide-co-e-caprolactone)/collagen nanoyarn network as a novel, threedimensional, macroporous, aligned scaffold for tendon tissue engineering Tissue Eng Part C Methods 19: 925-936.

13. Theiss F Mirsaidi A, Mhanna R, Kümmerle J Glanz S, et al. (2015) Use of biomimetic microtissue spheroids and specific growth factor supplementation to improve tenocyte differentiation and adaptation to a collagen-based scaffold in vitro. Biomaterials 69: 99-109.

14. Abou Neel EA Bozec L, Knowles JC, Syed O, Mudera V, et al. (2013) Collagen-emerging collagen based therapies hit the patient. Adv Drug Deliv Rev 65 429-456.

15. Oryan A, Silver IA, Goodship AE (2009) Effects of a serotonin S2-receptor blocker on healing of acute and chronic tendon injuries. J Invest Surg 22 246-255.

16. Liu CF, Aschbacher-Smith L, Barthelery NJ, Dyment N, Butler D, et al. (2011) What we should know before using tissue engineering techniques to repair injured tendons: a developmental biology perspective. Tissue Eng Part B Rev 17: $165-176$.

17. Del Buono A, Volpin A, Maffulli N (2014) Minimally invasive versus open surgery for acute Achilles tendon rupture: a systematic review. $\mathrm{Br}$ Med Bull 109: 45-54

18. Shapiro E, Grande D, Drakos M (2015) Biologics in Achilles tendon healing and repair: a review. Curr Rev Musculoskelet Med 8: 9-17.

19. Gross G, Hoffmann A (2013) Therapeutic strategies for tendon healing based on novel biomaterials, factors and cells. Pathobiology 80: 203-210.

20. Rodrigues MT, Reis RL, Gomes ME (2013) Engineering tendon and ligament tissues: present developments towards successful clinical products. J Tissue Eng Regen Med 7: 673-686. 
Citation: Oryan A, Alidadi S, Moshiri A (2015) Application of Collagen Implants in Achilles Tendon Injuries. J Sports Med Doping Stud 6: 169. doi:10.4172/2161-0673.1000169

Page 6 of 6

21. Moshiri A, Oryan A, Meimandiparizi A Maffulli N3 (2014) Collagen implants in experimental tendon injury in rabbits: a clinical, ultra-structural and biomechanical investigation. J Biol Regul Homeost Agents 28: 381-397.

22. Moshiri A, Oryan A, Meimandi-Parizi A, Silver IA, Tanideh N, et al. (2013) Effectiveness of hybridized nano- and microstructure biodegradable, biocompatible, collagen-based three-dimensional bioimplants in repair of a large tendon-defect model in rabbits. J Tissue Eng Regen Med. doi: 10.1002/ term. 1740 .

23. Chen JL, Yin Z, Shen WL, Chen X, Heng BC, et al. (2010) Efficacy of hESCMSCs in knitted silk-collagen scaffold for tendon tissue engineering and their roles. Biomaterials 31: 9438-9451.

24. Oryan A, Alidadi S, Moshiri A Maffulli N (2014) Bone regenerative medicine: classic options, novel strategies, and future directions. J Orthop Surg Res 9: 18.

25. Moshiri A, Oryan A, Meimandi-Parizi A, Koohi-Hosseinabadi O (2014) Effeciveness of xenogenous-based bovine-derived platelet gel embedded within a three-dimensional collagen implant on the Achilles tendon defect in rabbits. Expert Opin Biol Ther 14: 1065-1089.

26. Moshiri A, Oryan A, Meimandi-Parizi A (2015) Synthesis, development, characterization and effectiveness of bovine pure platelet gel-collagenpolydioxanone bioactive graft on tendon healing. J Cell Mol Med 19: 13081332.

27. Oryan A, Moshiri A, Parizi Meimandi A, Silver IA (2013) A long-term in vivo investigation on the effects of xenogenous based, electrospun, collagen implants on the healing of experimentally-induced large tendon defects. Musculoskelet Neuronal Interact 13: 353-367.

28. Oryan A, Moshiri A, Meimandi-Parizi A, Maffulli N (2015) Role of xenogenous bovine platelet gel embedded within collagen implant on tendon healing: an in vitro and in vivo study. Exp Biol Med (Maywood) 240: 194-210.

29. Zavatti M, Bertoni L, Maraldi T, Resca E, Beretti F, et al. (2015) Critical-size bone defect repair using amniotic fluid stem cell/collagen constructs: effect of oral ferutinin treatment in rats. Life Sci 121: 174-183.

30. Ratcliffe A, Butler DL, Dyment NA, Cagle PJ Jr, Proctor CS, et al. (2015) Scaffolds for tendon and ligament repair and regeneration. Ann Biomed Eng 43: 819-831.

31. Kunert-Keil C, Gredes T, Heinemann F, Dominiak M, Botzenhart U, et al. (2015) Socket augmentation using a commercial collagen-based product--an animal study in pigs. Mater Sci Eng C Mater Biol Appl 46: 177-183.

32. Zhao H, Guan HG, Gu J, Luo ZP, Zhang W, et al. (2013) Collagen membrane alleviates peritendinous adhesion in the rat Achilles tendon injury model. Chin Med J (Engl) 126: 729-733.

33. Sawadkar P, Alexander S, Mudera V (2014) Tissue-engineered collagen grafts to treat large tendon defects. Regen Med 9: 249-251.

34. Oryan A, Alidadi S, Moshiri A, Bigham-Sadegh A (2014) Bone morphogenetic proteins: a powerful osteoinductive compound with non-negligible side effects and limitations. Biofactors 40: 459-481.

35. Gomez-Guillen MC, Gimenez B, Lopez-Caballero ME (2011) Functional and bioactive properties of collagen and gelatin from alternative sources: A review. Food Hydrocoll 25: 1813-1827.
36. Wenpo F, Gaofeng L2, Shuying F3, Yuanming Q4, Keyong T5 (2015) Preparation and characterization of collagen-hydroxyapatite/pectin composite. Int J Biol Macromol 74: 218-223.

37. Krishnan AG, Jayaram L, Biswas R, Nair M (2015) Evaluation of antibacterial activity and cytocompatibility of ciprofloxacin loaded gelatin-hydroxyapatite scaffolds as a local drug delivery system for osteomyelitis treatment. Tissue Eng Part A 21: 1422-1431.

38. Hannink G, Geutjes PJ, Daamen WF, Buma P (2013) Evaluation of collagen/ heparin coated TCP/HA granules for long-term delivery of BMP-2. J Mater Sci Mater Med 24: 325-332.

39. Friess W (1998) Collagen--biomaterial for drug delivery. Eur J Pharm Biopharm 45: 113-136.

40. Moshiri A, Shahrezaee M, Shekarchi B, Oryan A, Azma K (2015) ThreeDimensional Porous Gelapin-Simvastatin Scaffolds Promoted Bone Defect Healing in Rabbits. Calcif Tissue Int 96: 552-564.

41. Kirchmajer DM, Watson CA, Ranson M, Panhuis M (2013) Gelapin, a degradable genipin cross-linked gelatin hydrogel. RSC Advances 3: 10731081

42. Nguyen TB, Min YK, Lee BT (2015) Nanoparticle biphasic calcium phosphate loading on gelatin-pectin scaffold for improved bone regeneration. Tissue Eng Part A 21: 1376-1387.

43. Kwon SY, Chung JW, Park HJ, Jiang YY, Park JK, et al. (2014) Silk and collagen scaffolds for tendon reconstruction. Proc Inst Mech Eng H 228 : 388-396.

44. Elder BD, Eleswarapu SV, Athanasiou KA (2009) Extraction techniques for the decellularization of tissue engineered articular cartilage constructs. Biomaterials 30: 3749-3756.

45. Vavken $P$, Joshi S, Murray MM (2009) TRITON-X is most effective among three decellularization agents for ACL tissue engineering. J Orthop Res 27: 1612-1618.

46. Pietschmann MF, Frankewycz B, Schmitz P, Docheva D, Sievers B, et al. (2013) Comparison of tenocytes and mesenchymal stem cells seeded on biodegradable scaffolds in a full-size tendon defect model. J Mater Sci Mater Med 24: 211-220.

47. Zhang W, Yang Y, Zhang K, Li Y, Fang G (2015) Weft-knitted silk-poly(lactideco-glycolide) mesh scaffold combined with collagen matrix and seeded with mesenchymal stem cells for rabbit Achilles tendon repair. Connect Tissue Res 56: 25-34.

48. Martinello T, Bronzini I, Volpin A, Vindigni V, Maccatrozzo L, et al. (2014) Successful recellularization of human tendon scaffolds using adipose-derived mesenchymal stem cells and collagen gel. J Tissue Eng Regen Med 8: 612619.

49. Liu H, Ding X, Zhou G, Li P, Xing Wei X, et al. (2013) Electrospinning of nanofibers for tissue engineering applications. J Nanomater 2013: 495708. 